\title{
Oxytocin effects on the resting-state mentalizing brain network
}

\author{
Haiyan $\mathrm{Wu}^{1,2,3}$, Chunliang Feng ${ }^{4}$, Xiaping $\mathrm{Lu}^{4}$, Xun Liu ${ }^{1,2}$ and Quanying Liu ${ }^{5,6,7 *}$ \\ 2 \\ 1 CAS Key Laboratory of Behavioral Science, Institute of Psychology, Beijing, China \\ 2 Department of Psychology, University of Chinese Academy of Sciences, Beijing, China \\ Pasadena, CA, USA \\ 4 State Key Laboratory of Cognitive Neuroscience and Learning, Beijing Normal University, China \\ 5 \\ 6 Neurosciences, Huntington Medical Research Institutes, Pasadena, CA, USA \\ 7 \\ Research Center for Motor Control and Neuroplasticity, KU Leuven, Leuven, Belgium
}

3

${ }^{*}$ Corresponding author:

Quanying Liu

Division of Engineering and Applied Science, California Institute of Technology,

Pasadena, CA, USA

Email: Quanying.Liu@caltech.edu 


\section{ABSTRACT}

Oxytocin(OT) has effects in both human behavior and in the brain, which is not limited in the specific brain area but also with the potential effect on connectivity with other brain regions. Evidence indicate that the effects of OT on human behavior are multifaceted, such as trust behavior, decrease anxiety, empathy and bonding behavior. Since the vital role of mentalizing in understanding others, here we proposed and tested that whether OT has a general effect on theory of mind brain network which is associated to the effect of related social behavioral and personality traits. Used a randomized, double-blind placebo-controlled group design, we investigated the resting-state functional magnetic resonance imaging after intranasal OT or placebo. The functional connectivity (FC) maps with seed in left temporoparietal junction (ITPJ) and right TPJ showed that OT significantly increased connectivity between rTPJ and default attention network (DAN), while decreased the FC between ITPJ and medial prefrontal network (MPN). With implementing machine learning approach, we further reported satisfactory classification accuracy that Identified altered FCs of TPJ can classify OT and PL group. Moreover, individual's empathy trait can modulate the FC between left TPJ and right RECT, which was positively correlated with empathic concern in PL group whereas ITPJ-rRECT negatively correlated in OT group. These results demonstrate that OT has significant effect on FC with ITPJ and rTPJ, brain regions critical for mentalizing, and the empathy concern can modulate the FC. These findings add to our understanding of the neural mechanisms by which OT modulates social behaviors, especially in social interaction involving mentalizing. 


\section{INTRODUCTION}

Oxytocin (OT) is a neuropeptide which has been shown to modulate complex emotional and social behaviors (Bartz et al., 2011), and mediate the relevant neural activity (Kirsch et al., 2005) and functional connectivity (Bethlehem et al., 2013; Dodhia et al., 2014). It has been evident that oxytocin modulates various social emotions and behaviors (Alvares et al., 2010; Hu et al., 2015; Kosfeld et al., 2005; Labuschagne et al., 2010a; Riem et al., 2011; Scheele et al., 2013; Shamay-Tsoory et al., 2009; Striepens et al., 2012; Theodoridou et al., 2009). For example, behavioral studies have shown that oxytocin administration induces more trust behaviors (Kosfeld et al., 2005), and decrease social distance from others (Preckel et al., 2014), and even more prosocial behaviors to social rejected others (Riem et al., 2013a). Neuroimaging studies have convergently shown oxytocin modulates brain activity in social behaviors (Wittfoth-Schardt et al., 2012), such as emotion recognition (Hurlemann et al., 2010), social memory (Rimmele et al., 2009) as well as prosocial behaviors (Mikolajczak et al., 2010). For instance, OT administration changes the brain activity in response to infant crying, including decreased the activity in amygdala and increased activity in both insula and inferior frontal gyrus (Riem et al., 2011), which may be attributed to increasing empathic responses. Similarly, OT-administrated subjects showed a reduced response of the right amygdala to angry, happy and fearful faces (Domes et al., 2007a). Accordingly, efforts have also been dedicated to its therapeutic potential in different kinds of social dysfunction disorders patients, in both behavioral and neural levels (Anagnostou et al., 2012; Labuschagne et al., 2010b; Modi and Young, 2012; Neumann and Landgraf, 2012). These results suggest that OT effect is specifically germane to social stimulus, and to the brain network activated by the stimulus.

While such attempts aim to understand the neurobiological basis of OT effect on social cognition through task-related functionally brain activities, there are growing interests in understanding brain network changes in task-free state, which are far less thoroughly investigated. To date, some studies in humans have investigated the effects of oxytocin on resting-state functional magnetic resonance imaging (rsfMRl). This body of work 
mainly motivated by hypothesis about the OT effect on emotion and amygdala pathway. For example, individuals with oxytocin misuse are with stronger connectivity between right amygdala and dorsal anterior cingulate cortex, relative to the control group (Kovacs and Keri, 2015). With a closer examination of amygdala subregions, Eckstein et al (2017) found OT exerts different amygdala subregion-specific connectivity patterns in resting state. In social anxiety disorder patients, oxytocin enhanced amygdala and rostral anterior cingulate cortex (ACC)/medial prefrontal cortex (mPFC) connectivity (Phan, 2014). Another related study also showed similar OT effect in healthy participants such that oxytocin significantly increased connectivity between amygdalae and rostral medial frontal cortex (Sripada et al., 2013). Of these studies investigating the effects of oxytocin on resting-state $\mathrm{fMRI}$ and connectivity, amygdala related brain network has been qualified to be affected by OT.

Although it has been reported that human oxytocin could modulate both social cognition and resting state brain connectivity, how oxytocin modulates other social brain networks engaged in these social behaviors (e.g., social inference, empathy and reward system), is still largely unclear. Notably, few study has focused on relationship between OT administration and mind-reading related brain network changes. Mind-reading is a process in which people draw internal states inference and the weight those inference with context information from external states (e.g. expressions, gestures, signals, etc.) (Realo et al., 2003). Large amounts of studies have shown that OT administration can improve mind-reading (Domes et al., 2007b; Radke and de Bruijn, 2015; Riem et al., 2014b; Voorthuis et al., 2014). For example, using the Reading the Mind in the Eyes Test (RMET), the accuracy was significantly improved after oxytocin administration (Domes et al., 2007c). One key cortical brain region involved in mind-reading functions is temporoparietal junction (TPJ) (Saxe and Wexler, 2005). Causal evidence showed that brain damage in this region could lead to dysfunctions in both moral decisions (Lamm et al., 2007) and empathy tasks (Benedetti et al., 2009; Cheon et al., 2011; Decety et al., 2008a; Dodell-Feder et al., 2011; Jackson et al., 2006; Lamm et al., 2011). Further evidence or meta-analysis showed separately functions in TPJ (Krall et al., 2015a; Kubit and Jack, 2013; Lombardo et al., 2011; Mitchell, 2008), however, another experimental 
design study showed support to the theory of mind hypothesis in TPJ (Young et al., 2010). Further, that TPJ connected brain has also been modulated by both empathy and social behaviors (Emonds et al., 2014). Clearly, most of the aforementioned studies show the connections between theory of mind, empathy and TPJ.

In light of those OT effect in mind reading, we speculate that the brain connectivity by which people perceive (perspective taking tasks) and infer about other's mind (theory of mind tasks) could be modulated by OT. Since previous neuroimaging studies have shown individual difference in OT effect on brain activity and its connectivity (Eckstein et al., 2017; Riem et al., 2014a; Riem et al., 2013b), we would also take into account of individual difference, such as empathy trait.

The Interpersonal Reactivity Index (IRI) (Davis, 1980; Davis, 1983) is a widely used scale to measure dispositional empathy. It has four subscales (i.e., perspective taking, fantasy, empathy concern, and personal distress). The perspective-taking scale assesses spontaneous attempts to adopt the perspectives of other people and see things from their point of view. Items on the fantasy scale measure the tendency to identify with characters in movies, novels, plays and other fictional situations. The other two subscales explicitly tap respondents' chronic emotional reactions to the negative experiences of others. It has been suggested that the mind-reading accuracy was increased after oxytocin administration for those lower in empathic concern scale of the IRI (Radke and de Bruijn, 2015). We thus measure the empathic trait of individual participant to tap into the possible moderation effect of empathetic trait on social brain network in terms of the resting state functional connectivity.

The aim of this study was to explore the effects of oxytocin on TPJ related functional connectivity in resting state. With the increasing concern about the individual difference and context effect in oxytocin effect on social cognition (Bartz et al., 2011; Churchland and Winkielman, 2012; Olff et al., 2013), we believe that the OT administration could lead to both broad influence of general state change and individual difference on resting brain network. Given prior evidence that oxytocin enhances mind reading accuracy and social salience hypothesis of oxytocin (Shamay-Tsoory and Abu-Akel, 2016), we hypothesized 
that OT administrated group would exhibit increased connectivity between TPJ to other social brain regions, relative to the group under placebo, and show moderation effect of individual personality traits.

\section{METHODS}

Participants

The study's participants included 59 right-handed male college students (age range 19 26 years, and education range 13 18 years), who were recruited via an online recruiting system. All participants filled out a screening form, and participants were included in the study only if they confirmed they were not suffering from any significant medical or psychiatric illness, not using medication, not drinking and/or smoking in a daily basis. Participants were instructed to refrain from smoking or drinking (except water) for 2 hours before the experiment. All experiments were conducted in Beijing Normal University. Participants received full debriefing on completion of the experiment. All participants provided written consent, and the study protocol was approved by Institutional Review Board (IRB) of Beijing Normal University.

\section{Study Design and Drug Administration}

We used a double-blind placebo-controlled group design to investigate the effects of a single dose of intranasal oxytocin on the resting-state functional connectivity. Participants randomly assigned to oxytocin (OT) group or the placebo (PL) group (Table 1). Each participant visited twice, with the first visit to fill in questionnaires (e.g., the Interpersonal Reactivity Index (IRI)) and the second visit for MRI scanning.

Each participant arrived for the second visit 1 hour before MRI scanning. Participants did not consume food, caffeine or alcohol 1 hour before the MRI scanning. At 45 minutes before the MRI scanning, a single dose of $24 \mathrm{IU}$ oxytocin or placebo was administered intranasally by three puffs of $8 \mathrm{IU}$ per nostril. The puffs were delivered to each nostril in an alternating order and with a 45-second break between each puff to each nostril. The participants have 5-minute resting-state scan before any tasks. Each subject was positioned supine in the MRI scanner with his head in a comfortable restraint to reduce 
movement. Subjects were instructed to keep their eyes closed, relax and let their minds wander, but not to fall sleep. Following the resting-state scan, participants performed $\mathrm{N}$ different cognitive tasks. These task-relevant data were analyzed separately from the resting-state results.

\section{MRI Acquisition}

A 3T Siemens Tim Trio scanner with 12-channel head coil was used to perform MRI. Functional images employed a gradient-echo echo-planar imaging (EPI) sequence that is sensitive to blood oxygenated level-dependent (BOLD) contrast (40 ms TE, $2 \mathrm{~s}$ TR, 90 flip, $210 \mathrm{~mm}$ FOV, $128 \times 128$ matrix, 25 contiguous $5 \mathrm{~mm}$ slices parallel to the hippocampus and interleaved). We also acquired from all participants whole-brain T1weighed anatomical reference images (2.15 ms TE, $1.9 \mathrm{~s}$ TR, $9^{\circ} \mathrm{flip}, 256 \mathrm{~mm}$ FOV, 176 sagittal slices, $1 \mathrm{~mm}$ slice thickness, perpendicular to the anterior-posterior commissure line).

\section{Data preprocessing}

$\mathrm{fMRI}$ data preprocessing was performed using Statistical Parametric Mapping software (SPM12: Wellcome Trust Centre for Neuroimaging, London, UK). The functional image time series were preproessed to compensate for slice-dependent time shifts, motion corrected, and linearly detrended, then coregistered to the anatomical image, spatial normalized to Montreal Neurological Institute (MNI) space (http://www.bic.mni.mcgill.ca/ServicesAtlases/HomePage) and spatially smoothed by convolution with an isotropic Gaussian kernel (FWHM=6 mm). The fMRI data were highpass filtered with a cutoff of $0.01 \mathrm{~Hz}$. The white matter (WM) signal, cerebrospinal fluid (CSF) signal and global signal, as well as the 6-dimensional head motion realignment parameters, the realignment parameters squared, their derivatives, and the squared of the derivatives were regressed. The resulting residuals were then low-pass filtered with a cutoff of $0.1 \mathrm{~Hz}$.

ICA-based RSN extraction

We used a data-driven approach to extract RSNs from both OT and PL groups, to investigate the effects of OT on large-scale functional networks. Specifically, 14 regular 
RSNs were extracted in each individual fMRI dataset by using ICA-based approach(Beckmann et al., 2009) , combining a template matching strategy (Liu et al., 2017), including cingulo-opercular network (CON), default mode network (DMN), dorsal attention network (DAN), dorsal somatomotor network (DSN), auditory network (AN), language network (LN), left frontoparietal network (IFPN), right frontoparietal network (rFPN), medial prefrontal network (MPN) and lateral prefrontal network (LPN), ventral attention network (VAN), ventral somatomotor network (VSN), visual foveal network (VFN) and visual peripheral network (VPN). The group-level RSN maps for both groups were obtained by using a voxel-wise non-parametric permutation test by FSL (http://fsl.fmrib.ox.ac.uk/fsl/fslwiki) with 5000 permutations. Besides, two-sample unpaired t-test between OT and PL group was applied to investigate the topographic changes induced by OT.

Seed-based functional connectivity analysis

The analysis pipeline was conducted in MATLAB and was similar to that employed in our previous research (Wu et al., 2015). We averaged the signals in left and right TPJ masks, which were defined by previous literature (Alcala-Lopez et al., 2017). The temporal correlations between seed region and the rest of the brain were examined, subject by subject. To generate the TPJ connectivity map for each group, one-sample t-tests were performed for both OT and PL group by running randomise function in FSL. To compare the FC between OT and PL group, two-sample unpaired t-test was applied. The resulting maps were thresholded at $p<0.05$ with threshold-free cluster enhancement (TFCE) correction (Smith and Nichols, 2009). The clustered regions with peak MNI coordinates were exported by cluster function in FSL. We used the FCs between TPJ and these identified regions for the following analyses.

\section{Classification of OT and PL with TPJ functional connectivity}

To examine whether the distinction the TPJ connectivity patterns from OT and PL were consistent across participants, we used a linear support vector machine (SVM) classifier and the identified FC as input features to distinguish OT and PL. SVM identifies the weight 
of each FC that define a maximum margin hyperplane that best separates two classes of data (Dosenbach et al., 2010). We used leave-one-out cross-validation (LOOCV), where the classifier was trained on 58 participants (svmtrain function in MATLAB), then tested on the remaining one participant (svmclassify function in MATLAB). The performance was quantified using the accuracy rate, sensitivity and specificity based on the results of crossvalidation (Zeng et al., 2012). The weight of each FC was recorded for all iterations of the cross-validation to examine the reliability of consensus functional connectivity discriminative power.

Post-hoc correlation analyses

To investigate whether the participant's dispositional empathy intermediate the oxytocin effect on TPJ connectivity, we calculated the partial correlation between each IRI subscale and FCs of the identified brain regions, by statistically removing the other subscales. The false discovery rate (FDR) correction were performed on $p$ values.

\section{RESULTS}

Oxytocin effects on TPJ brain connectivity

We firstly obtained the FC maps with seed in left TPJ and right TPJ and examine the group-level FC maps in OT group and PL group, respectively (Figure 1). Clearly, we found that TPJ positively connected with the brain regions involved in default mode network (DMN) and medial prefrontal network (MPN), but negatively correlated with the brain regions in dorsal attention network (DAN). To quantify the oxytocin effects on the functional connectivity between TPJ and brain networks, we extracted 14 resting-state networks (RSNs) by ICA approach (supplementary Figure 1). Surprisingly, no topological differences of RSNs from OT and PL group were significant, which indicated that oxytocin does not change the topography of RSNs. We further calculate the FC between TPJ and 14 extracted RSNs, and proved that both left and right TPJ strongly synchronized with DMN and VAN, and anticorrelated with CON, DSN and AN (Figure 2A and 2B). 
Moreover, compared with PL group, the FC between ITPJ and MPN in OT is significantly weaker $(\mathrm{t}=-3.64, \mathrm{p}=0.0083)$, whereas $\mathrm{rTPJ}$-DAN in OT group is significant stronger $(t=3.84, p=0.0044)$. All the reported $p$ values were FDR corrected.

Identified altered FCs

To compare the OT effect with placebo effect, we performed the between-group comparison (Figure 1). The resulting maps identified 8 brain regions $(p<0.05$, TFCE corrected; Table 1). We averaged the BOLD signals in these regions and then calculated the Pearson correlation between the identified brain regions and the corresponding seed region. Thus, we obtained 8 pairs of FCs with significant OT effects (ps<0.001, twosample t-test), including ITPJ-IIPL ( $r=4.52)$, rTPJ-IPrec $(r=5.36)$, rTPJ-rSPL $(r=3.90)$, rTPJ-rPrec ( $r=3.82)$, rTPJ-IMOG ( $r=3.96)$ where the connectivity is higher in OT group, and ITPJ-rRECT ( $r=-4.35)$, rTPJ-IInsula $(r=-4.11)$, rTPJ-IIFG $(r=-4.12)$ where in intranasal OT reduced the coupling (Supplementary Figure 2).

SVM classification based on the identified FCs

Using the identified eight FCs, the accuracy of classification was $74.58 \%$ with sensitivity of $73.33 \%$ and specificity of $75.86 \%$. Moreover, the weights of input features were generated for each LOOCV training, which represents the contribution of each FC in classification, with a larger weight indicating larger contribution. Our results showed that ITPJ-rRect connectivity has the highest contribution. Intriguingly, the weights of these FCs were quite robust across subjects (see the mean and standard error of weights in Table 2), which indicates the reliable properties of TPJ related FC changes induced by OT administration.

IRI intermediates the oxytocin effects on FC

To disentangle the influence of personal empathy on oxytocin effects, we calculated the partial correlation between each IRI subscale whilst controlling for the effect of other subscales. We found that the FC between left TPJ and right RECT positively correlated with empathic concern in PL group ( $r=0.47, p=0.059$, Figure $4 A$ ), whereas ITPJ-rRECT 
negatively correlated in OT group $(r=-0.51, p=0.029$, Figure 4B). No FCs showed significant correlation with perspective taking, fantasy and personal distress.

\section{DISCUSSION}

Even playing a key role in social cognition, activity and connectivity of social brain networks in task-free state under oxytocin administration, are still not well understood. Recent studies have investigated amygdala -related brain network after OT administration. However, there are still other functions and brain networks relevant to social cognition that could give rise to these effects, which were not well investigated in previous work. In order to assess the OT effect on properties of mentalizing related brain networks, we examined OT effects on TPJ brain connectivity on putative brain networks and on social brain networks. The OT induced brain connectivity in social brain networks can be classified by distinguished FCs, which is modulated by individual traits.

Interactions between TPJ and RSNs

Our results suggest that TPJ is generally positively correlated with DMN and MPN, but negatively correlated with DAN, independent of drug administration. The DMN is supposed to be the brain areas that are typically more active during rest state than during active task performance. However, it has been observed that DMN is partly overlapping with the social brain (Mars et al., 2012; Schilbach et al., 2008; Spreng et al., 2009; Washington et al., 2014; Xie et al., 2016). Our findings add further evidence on it based on the overlap between ICA-identified DMN and MPN (Figure 1). In line with the concept that TPJ connection with MPFC is vital in the understanding of other's mental states (Li et al., 2014), we believe the observed generic positive connection between TPJ and MPN is related to monitor both self and other mental states. The negative connection between TPJ and DAN we observed, appears to echo existing findings with a dissociated effect on the DAN and ventral attention network (VAN) (Fox et al., 2006). It has been shown that the DAN is engaged in top-down orienting, while VAN activates for bottom-up salience detection (Corbetta and Shulman, 2002). As the TPJ acts as a part of VAN (Corbetta et 
al., 2000), the negative correlation reflects spontaneous neuronal activity between the two attention system (VAN and DAN) are relatively deviated (Fox et al., 2006). For example, previous work has shown decreased TPJ activity when cognitive demands are imposed and the DAN is engaged (Shulman et al., 2003).

OT effects on TPJ functional connectivity

It is interesting that oxytocin does not change the ICA-identified topography of RSNs, but changes the FCs between TPJ and RSNs. Specifically, the PL group had stronger left TPJ and MPN connection, whereas OT group showed stronger connection between right TPJ and DAN. In accordance with our initial hypotheses that TPJ connection may play an essential role in self-other mental state monitoring process, the weaker connection in the PL group may due to the less sensitivity of social signal. Conversely, the stronger connection between right TPJ and DAN in OT group may be related to the enforced social attention, which provide the support for social salience hypothesis of oxytocin. Social inference and attention functions are considered as two aspects of function in TPJ (Krall et al., 2015b), where is also brain area that attention and memory information converged (Carter and Huettel, 2013). Since attention or working memory studies showing causality activity between VAN and DAN (Sridharan et al., 2007) and the essential role of right TPJ in theory of mind (Hooker et al., 2010; Young et al., 2010), the enhancing rTPJ and DAN connection might be explained by increased social perception function.

Importantly, it seems that the significant FC differences between PL and OT group provides further evidence of the social attention hypothesis. In particular, right TPJ connection with nearby brain regions such as precuneus, inferior parietal lobule, and superior parietal lobule, is stronger in OT group. These brain regions are proposed to play critical role in salient detection or attention sustaining(Singh-Curry and Husain, 2009). Such results are also in line with previous studies reported increased functional connectivity between TPJ and near parietal brain areas in functional connectivity analyses (Decety et al., 2008b; Igelstrom and Graziano, 2017).

FC on TPJ allows to classify OT and PL group 
SVM classification with the resting-state FCs has been largely used for detecting brain diseases (Zeng et al., 2012), predicting brain maturity(Dosenbach et al., 2010), or mental states (Tagliazucchi and Laufs, 2014). Here we adopt it to investigate whether the TPJ related functional connectivity allows to robustly differentiate OT and PL group at the individual level. Impressively, using only eight most discriminative FCs, rather than a large number of FC features, can successfully detect $74.58 \%$ of participants. It confirms that these FC changes are caused by OT effect, and the effects are reliable at individual level. FC between ITPJ and rRect has highest contribution to the classification, as the connection is higher in PL group than OT group. Existing network efficiency analysis finding showed decreased connection strength between right temporal, parietal and occipital lobes in high-risk of autism spectrum disorder (ASD), without report stronger connection in ASD (Lewis et al., 2014). Combining the findings of increased ITPJ-IIPL, rTPJ-IPrec, rTPJ-rSPL, rTPJ-rPrec, our finding may indicate a OT effect of strengthen ipsilateral brain connection, especially from the right TPJ, while weaken contralateral brain connection from left TPJ.

\section{Empathic concern intermediates OT effects on FC}

Our results show that drug has an interaction effect on the FC between TPJ and Rectus (see Figure 4). Specifically, ITPJ-RECT resting state connectivity positively correlated with empathic concern in PL group. Using task related fMRI, previous study has shown that brain activity in ventral MPFC, including gyrus rectus, positively correlated with empathic ability (Schulte-Ruther et al., 2011). Also, it has been reported that TPJ activity positively correlated with the ingroup empathy bias (Cheon et al., 2011). Considering these findings are based on OT-free subjects, they explain the positive correlation between TPJ-rectus FC and empathic concern in PL group but not in OT group.

\section{Limitation and future work}

Potential limitation and future work should be recognized. A large limit of this study is that we did not collect resting $\mathrm{fMRI}$ data before and after the OT administration, thus this study 
cannot directly show a causal effect of OT on functional connectivity. Future work with the comparisons from the same group of subjects should be conducted. Moreover, ICA cleanup in data preprocessing, such as FIX implemented in FSL (Salimi-Khorshidi et al., 2014), can be applied in the future to remove noise effects before FC analyses. Another limitation of this work is that the input features for SVM are identified from FC images, and we did not perform any type of feature selection during training procedure. Filter methods or wrapper methods to select optimal FC features (Kassraian-Fard et al., 2016) from a large FC pool might be potential way to further improve the accuracy of classification.

\section{ACKNOWLEDGEMENT}

This work was supported by the National Natural Science Foundation of China [grant number: U1736125, 31400963], and CAS Key Laboratory of Behavioral Science, Institute of Psychology to HW. QL was supported by James Boswell fellowship and FWO fellowship.

\section{CONFLICT OF INTEREST}

The authors declare no competing financial interests. 
Alcala-Lopez, D., Smallwood, J., Jefferies, E., Van Overwalle, F., Vogeley, K., Mars, R.B., Turetsky, B.I., Laird, A.R., Fox, P.T., Eickhoff, S.B., Bzdok, D., 2017. Computing the Social Brain Connectome Across Systems and States. Cereb Cortex, 1-26.

Alvares, G.A., Hickie, I.B., Guastella, A.J., 2010. Acute effects of intranasal oxytocin on subjective and behavioral responses to social rejection. Exp Clin Psychopharmacol 18, 316-321. Anagnostou, E., Soorya, L., Chaplin, W., Bartz, J., Halpern, D., Wasserman, S., Wang, A.T., Pepa, L., Tanel, N., Kushki, A., Hollander, E., 2012. Intranasal oxytocin versus placebo in the treatment of adults with autism spectrum disorders: a randomized controlled trial. Molecular Autism 3.

Bartz, J.A., Zaki, J., Bolger, N., Ochsner, K.N., 2011. Social effects of oxytocin in humans: context and person matter. Trends in Cognitive Sciences 15, 301-309.

Beckmann, C.F., Mackay, C.E., Filippini, N., Smith, S.M., 2009. Group comparison of restingstate FMRI data using multi-subject ICA and dual regression. Neuroimage 47, S148.

Benedetti, F., Bernasconi, A., Bosia, M., Cavallaro, R., Dallaspezia, S., Falini, A., Poletti, S., Radaelli, D., Riccaboni, R., Scotti, G., Smeraldi, E., 2009. Functional and structural brain correlates of theory of mind and empathy deficits in schizophrenia. Schizophrenia Research 114, 154-160.

Bethlehem, R.A.I., van Honk, J., Auyeung, B., Baron-Cohen, S., 2013. Oxytocin, brain physiology, and functional connectivity: A review of intranasal oxytocin fMRI studies. Psychoneuroendocrinology 38, 962-974.

Carter, R.M., Huettel, S.A., 2013. A nexus model of the temporal-parietal junction. Trends in Cognitive Sciences 17, 328-336.

Cheon, B.K., Im, D.M., Harada, T., Kim, J.S., Mathur, V.A., Scimeca, J.M., Parrish, T.B., Park, H.W., Chiao, J.Y., 2011. Cultural influences on neural basis of intergroup empathy. Neuroimage 57, 642-650.

Churchland, P.S., Winkielman, P., 2012. Modulating social behavior with oxytocin: How does it work? What does it mean? Hormones and Behavior 61, 392-399.

Corbetta, M., Kincade, J.M., Ollinger, J.M., McAvoy, M.P., Shulman, G.L., 2000. Voluntary orienting is dissociated from target detection in human posterior parietal cortex (vol 3, pg 292, 2000). Nature Neuroscience 3, 521-521.

Corbetta, M., Shulman, G.L., 2002. Control of goal-directed and stimulus-driven attention in the brain. Nat Rev Neurosci 3, 201-215.

Davis, M.H., 1980. A multidimensional approach to individual differences in empathy. Davis, M.H., 1983. Measuring Individual-Differences in Empathy - Evidence for a Multidimensional Approach. Journal of Personality and Social Psychology 44, 113-126.

Decety, J., Michalska, K.J., Akitsuki, Y., 2008a. Who caused the pain? An fMRI investigation of empathy and intentionality in children. Neuropsychologia 46, 2607-2614.

Decety, J., Michalska, K.J., Akitsuki, Y., 2008b. Who caused the pain? An fMRI investigation of empathy and intentionality in children. Neuropsychologia 46, 2607-2614.

Dodell-Feder, D., Koster-Hale, J., Bedny, M., Saxe, R., 2011. fMRI item analysis in a theory of mind task. Neuroimage 55, 705-712.

Dodhia, S., Hosanagar, A., Fitzgerald, D.A., Labuschagne, I., Wood, A.G., Nathan, P.J., Phan, K.L., 2014. Modulation of Resting-State Amygdala-Frontal Functional Connectivity by Oxytocin in Generalized Social Anxiety Disorder. Neuropsychopharmacology 39, 2061-2069. 
Domes, G., Heinrichs, M., Glascher, J., Buchel, C., Braus, D.F., Herpertz, S.C., 2007a. Oxytocin attenuates amygdala responses to emotional faces regardless of valence. Biological Psychiatry 62, 1187-1190.

Domes, G., Heinrichs, M., Michel, A., Berger, C., Herpertz, S.C., 2007b. Oxytocin improves "mind-reading" in humans. Biological Psychiatry 61, 731-733.

Domes, G., Heinrichs, M., Michel, A., Berger, C., Herpertz, S.C., 2007c. Oxytocin improves "mind-reading" in humans. Biol Psychiatry 61, 731-733.

Dosenbach, N.U.F., Nardos, B., Cohen, A.L., Fair, D.A., Power, J.D., Church, J.A., Nelson, S.M., Wig, G.S., Vogel, A.C., Lessov-Schlaggar, C.N., Barnes, K.A., Dubis, J.W., Feczko, E., Coalson, R.S., Pruett, J.R., Barch, D.M., Petersen, S.E., Schlaggar, B.L., 2010. Prediction of Individual Brain Maturity Using fMRI. Science 329, 1358-1361.

Eckstein, M., Markett, S., Kendrick, K.M., Ditzen, B., Liu, F., Hurlemann, R., Becker, B., 2017. Oxytocin differentially alters resting state functional connectivity between amygdala subregions and emotional control networks: Inverse correlation with depressive traits. Neuroimage 149, 458-467.

Emonds, G., Declerck, C.H., Boone, C., Seurinck, R., Achten, R., 2014. Establishing cooperation in a mixed-motive social dilemma. An fMRI study investigating the role of social value orientation and dispositional trust. Soc Neurosci 9, 10-22.

Fox, M.D., Corbetta, M., Snyder, A.Z., Vincent, J.L., Raichle, M.E., 2006. Spontaneous neuronal activity distinguishes human dorsal and ventral attention systems (vol 103, pg 10046, 2006). Proceedings of the National Academy of Sciences of the United States of America 103, 1356013560.

Hooker, C.I., Verosky, S.C., Germine, L.T., Knight, R.T., D'Esposito, M., 2010. Neural activity during social signal perception correlates with self-reported empathy. Brain Res 1308, 100-113. Hu, J., Qi, S., Becker, B., Luo, L., Gao, S., Gong, Q., Hurlemann, R., Kendrick, K.M., 2015. Oxytocin selectively facilitates learning with social feedback and increases activity and functional connectivity in emotional memory and reward processing regions. Hum Brain Mapp 36, 2132-2146.

Hurlemann, R., Patin, A., Onur, O.A., Cohen, M.X., Baumgartner, T., Metzler, S., Dziobek, I., Gallinat, J., Wagner, M., Maier, W., Kendrick, K.M., 2010. Oxytocin enhances amygdaladependent, socially reinforced learning and emotional empathy in humans. J Neurosci 30, 49995007.

Igelstrom, K.M., Graziano, M.S.A., 2017. The inferior parietal lobule and temporoparietal junction: A network perspective. Neuropsychologia 105, 70-83.

Jackson, P.L., Brunet, E., Meltzoff, A.N., Decety, J., 2006. Empathy examined through the neural mechanisms involved in imagining how I feel versus how you feel pain.

Neuropsychologia 44, 752-761.

Kassraian-Fard, P., Matthis, C., Balsters, J.H., Maathuis, M.H., Wenderoth, N., 2016. Promises, Pitfalls, and Basic Guidelines for Applying Machine Learning Classifiers to Psychiatric Imaging data, with Autism as an example. Frontiers in Psychiatry 7.

Kirsch, P., Esslinger, C., Chen, Q., Mier, D., Lis, S., Siddhanti, S., Gruppe, H., Mattay, V.S., Gallhofer, B., Meyer-Lindenberg, A., 2005. Oxytocin modulates neural circuitry for social cognition and fear in humans. Journal of Neuroscience 25, 11489-11493.

Kosfeld, M., Heinrichs, M., Zak, P.J., Fischbacher, U., Fehr, E., 2005. Oxytocin increases trust in humans. Nature 435, 673-676. 
Kovacs, B., Keri, S., 2015. Off-label intranasal oxytocin use in adults is associated with increased amygdala-cingulate resting-state connectivity. European Psychiatry 30, 542-547. Krall, S.C., Rottschy, C., Oberwelland, E., Bzdok, D., Fox, P.T., Eickhoff, S.B., Fink, G.R., Konrad, K., 2015a. The role of the right temporoparietal junction in attention and social interaction as revealed by ALE meta-analysis. Brain Structure \& Function 220, 587-604. Krall, S.C., Rottschy, C., Oberwelland, E., Bzdok, D., Fox, P.T., Eickhoff, S.B., Fink, G.R., Konrad, K., 2015b. The role of the right temporoparietal junction in attention and social interaction as revealed by ALE meta-analysis. Brain Struct Funct 220, 587-604.

Kubit, B., Jack, A.I., 2013. Rethinking the role of the rTPJ in attention and social cognition in light of the opposing domains hypothesis: findings from an ALE-based meta-analysis and resting-state functional connectivity. Front Hum Neurosci 7, 323.

Labuschagne, I., Phan, K.L., Wood, A., Angstadt, M., Chua, P., Heinrichs, M., Stout, J.C., Nathan, P.J., 2010a. Oxytocin Attenuates Amygdala Reactivity to Fear in Generalized Social Anxiety Disorder. Neuropsychopharmacology 35, 2403-2413.

Labuschagne, L., Phan, K.L., Wood, A., Chua, P., Angstadt, M., Heinrichs, M., Stout, J., Nathan, P.J., 2010b. Oxytocin Attenuates Amygdala Response to Fear in Social Anxiety Disorder. Biological Psychiatry 67, 203s-203s.

Lamm, C., Decety, J., Singer, T., 2011. Meta-analytic evidence for common and distinct neural networks associated with directly experienced pain and empathy for pain. Neuroimage 54, 24922502.

Lamm, C., Nusbaum, H.C., Meltzoff, A.N., Decety, J., 2007. What are you feeling? Using functional magnetic resonance imaging to assess the modulation of sensory and affective responses during empathy for pain. PLoS One 2, e1292.

Lewis, J.D., Evans, A.C., Pruett, J.R., Botteron, K., Zwaigenbaum, L., Estes, A., Gerig, G., Collins, L., Kostopoulos, P., McKinstry, R., Dager, S., Paterson, S., Schultz, R.T., Styner, M., Hazlett, H., Piven, J., Network, I., 2014. Network inefficiencies in autism spectrum disorder at 24 months. Translational Psychiatry 4.

Li, W.Q., Mai, X.Q., Liu, C., 2014. The default mode network and social understanding of others: what do brain connectivity studies tell us. Frontiers in Human Neuroscience 8.

Liu, Q.Y., Farahibozorg, S., Porcaro, C., Wenderoth, N., Mantini, D., 2017. Detecting Large-

Scale Networks in the Human Brain Using High-Density Electroencephalography. Human Brain Mapping 38, 4631-4643.

Lombardo, M.V., Chakrabarti, B., Bullmore, E.T., Consortium, M.A., Baron-Cohen, S., 2011. Specialization of right temporo-parietal junction for mentalizing and its relation to social impairments in autism. Neuroimage 56, 1832-1838.

Mars, R.B., Neubert, F.X., Noonan, M.P., Sallet, J., Toni, I., Rushworth, M.F.S., 2012. On the relationship between the "default mode network" and the "social brain". Frontiers in Human Neuroscience 6.

Mikolajczak, M., Gross, J.J., Lane, A., Corneille, O., de Timary, P., Luminet, O., 2010. Oxytocin makes people trusting, not gullible. Psychological Science 21, 1072-1074.

Mitchell, J.P., 2008. Activity in right temporo-parietal junction is not selective for theory-ofmind. Cerebral Cortex 18, 262-271.

Modi, M.E., Young, L.J., 2012. The oxytocin system in drug discovery for autism: Animal models and novel therapeutic strategies. Horm Behav 61, 340-350.

Neumann, I.D., Landgraf, R., 2012. Balance of brain oxytocin and vasopressin: implications for anxiety, depression, and social behaviors. Trends Neurosci 35, 649-659. 
Olff, M., Frijling, J.L., Kubzansky, L.D., Bradley, B., Ellenbogen, M.A., Cardoso, C., Bartz, J.A., Yee, J.R., van Zuiden, M., 2013. The role of oxytocin in social bonding, stress regulation and mental health: An update on the moderating effects of context and interindividual differences. Psychoneuroendocrinology 38, 1883-1894.

Phan, K.L., 2014. Oxytocin Modulation of Amygdala-Frontal Reactivity and Connectivity to Threat and at Rest in Social Anxiety Disorder. Biological Psychiatry 75, 27s-27s.

Preckel, K., Scheele, D., Kendrick, K.M., Maier, W., Hurlemann, R., 2014. Oxytocin facilitates social approach behavior in women. Frontiers in Behavioral Neuroscience 8, 191.

Radke, S., de Bruijn, E.R.A., 2015. Does oxytocin affect mind-reading? A replication study. Psychoneuroendocrinology 60, 75-81.

Realo, A., Allik, J., Nõlvak, A., Valk, R., Ruus, T., Schmidt, M., Eilola, T., 2003. Mind-reading ability: Beliefs and performance. Journal of Research in Personality 37, 420-445.

Riem, M.M., Bakermans-Kranenburg, M.J., Huffmeijer, R., van Ijzendoorn, M.H., 2013a. Does intranasal oxytocin promote prosocial behavior to an excluded fellow player? A randomizedcontrolled trial with Cyberball. Psychoneuroendocrinology 38, 1418-1425.

Riem, M.M., Bakermans-Kranenburg, M.J., Pieper, S., Tops, M., Boksem, M.A., Vermeiren, R.R., van Ijzendoorn, M.H., Rombouts, S.A., 2011. Oxytocin modulates amygdala, insula, and inferior frontal gyrus responses to infant crying: a randomized controlled trial. Biol Psychiatry 70, 291-297.

Riem, M.M., Bakermans-Kranenburg, M.J., Voorthuis, A., van, I.M.H., 2014a. Oxytocin effects on mind-reading are moderated by experiences of maternal love withdrawal: an fMRI study. Prog Neuropsychopharmacol Biol Psychiatry 51, 105-112.

Riem, M.M., van, I.M.H., Tops, M., Boksem, M.A., Rombouts, S.A., Bakermans-Kranenburg, M.J., 2013b. Oxytocin effects on complex brain networks are moderated by experiences of maternal love withdrawal. Eur Neuropsychopharmacol 23, 1288-1295.

Riem, M.M.E., Bakermans-Kranenburg, M.J., Voorthuis, A., van IJzendoorn, M.H., $2014 b$. Oxytocin effects on mind-reading are moderated by experiences of maternal love withdrawal: An fMRI study. Progress in Neuro-Psychopharmacology \& Biological Psychiatry 51, 105-112. Rimmele, U., Hediger, K., Heinrichs, M., Klaver, P., 2009. Oxytocin makes a face in memory familiar. J Neurosci 29, 38-42.

Salimi-Khorshidi, G., Douaud, G., Beckmann, C.F., Glasser, M.F., Griffanti, L., Smith, S.M., 2014. Automatic denoising of functional MRI data: combining independent component analysis and hierarchical fusion of classifiers. Neuroimage 90, 449-468.

Saxe, R., Wexler, A., 2005. Making sense of another mind: the role of the right temporo-parietal junction. Neuropsychologia 43, 1391-1399.

Scheele, D., Wille, A., Kendrick, K.M., Stoffel-Wagner, B., Becker, B., Gunturkun, O., Maier, W., Hurlemann, R., 2013. Oxytocin enhances brain reward system responses in men viewing the face of their female partner. Proc Natl Acad Sci U S A 110, 20308-20313.

Schilbach, L., Eickhoff, S.B., Rska-Jagiela, A.R., Fink, G.R., Vogeley, K., 2008. Minds at rest? Social cognition as the default mode of cognizing and its putative relationship to the "default system" of the brain. Consciousness and Cognition 17, 457-467.

Schulte-Ruther, M., Greimel, E., Markowitsch, H.J., Kamp-Becker, I., Remschmidt, H., Fink, G.R., Piefke, M., 2011. Dysfunctions in brain networks supporting empathy: an fMRI study in adults with autism spectrum disorders. Soc Neurosci 6, 1-21.

Shamay-Tsoory, S.G., Abu-Akel, A., 2016. The Social Salience Hypothesis of Oxytocin. Biological Psychiatry 79, 194-202. 
Shamay-Tsoory, S.G., Fischer, M., Dvash, J., Harari, H., Perach-Bloom, N., Levkovitz, Y., 2009. Intranasal administration of oxytocin increases envy and schadenfreude (gloating). Biol Psychiatry 66, 864-870.

Shulman, G.L., McAvoy, M.P., Cowan, M.C., Astafiev, S.V., Tansy, A.P., d'Avossa, G., Corbetta, M., 2003. Quantitative analysis of attention and detection signals during visual search. Journal of Neurophysiology 90, 3384-3397.

Singh-Curry, V., Husain, M., 2009. The functional role of the inferior parietal lobe in the dorsal and ventral stream dichotomy. Neuropsychologia 47, 1434-1448.

Smith, S.M., Nichols, T.E., 2009. Threshold-free cluster enhancement: Addressing problems of smoothing, threshold dependence and localisation in cluster inference. Neuroimage 44, 83-98. Spreng, R.N., Mar, R.A., Kim, A.S.N., 2009. The Common Neural Basis of Autobiographical Memory, Prospection, Navigation, Theory of Mind, and the Default Mode: A Quantitative Metaanalysis. Journal of Cognitive Neuroscience 21, 489-510.

Sridharan, D., Levitin, D.J., Chafe, C.H., Berger, J., Menon, V., 2007. Neural dynamics of event segmentation in music: converging evidence for dissociable ventral and dorsal networks. Neuron 55, 521-532.

Sripada, C.S., Phan, K.L., Labuschagne, I., Welsh, R., Nathan, P.J., Wood, A.G., 2013. Oxytocin enhances resting-state connectivity between amygdala and medial frontal cortex. International Journal of Neuropsychopharmacology 16, 255-260.

Striepens, N., Scheele, D., Kendrick, K.M., Becker, B., Schafer, L., Schwalba, K., Reul, J., Maier, W., Hurlemann, R., 2012. Oxytocin facilitates protective responses to aversive social stimuli in males. Proc Natl Acad Sci U S A 109, 18144-18149.

Tagliazucchi, E., Laufs, H., 2014. Decoding Wakefulness Levels from Typical fMRI RestingState Data Reveals Reliable Drifts between Wakefulness and Sleep. Neuron 82, 695-708. Theodoridou, A., Rowe, A.C., Penton-Voak, I.S., Rogers, P.J., 2009. Oxytocin and social perception: oxytocin increases perceived facial trustworthiness and attractiveness. Horm Behav $56,128-132$.

Voorthuis, A., Riem, M.M.E., Van Ijzendoorn, M.H., Bakermans-Kranenburg, M.J., 2014. Reading the mind in the infant eyes: Paradoxical effects of oxytocin on neural activity and emotion recognition in watching pictures of infant faces. Brain Research 1580, 151-159.

Washington, S.D., Gordon, E.M., Brar, J., Warburton, S., Sawyer, A.T., Wolfe, A., MeaseFerence, E.R., Girton, L., Hailu, A., Mbwana, J., Gaillard, W.D., Kalbfleisch, M.L., VanMeter, J.W., 2014. Dysmaturation of the Default Mode Network in Autism. Human brain mapping 35, 1284-1296.

Wittfoth-Schardt, D., Grunding, J., Wittfoth, M., Lanfermann, H., Heinrichs, M., Domes, G., Buchheim, A., Gundel, H., Waller, C., 2012. Oxytocin Modulates Neural Reactivity to Children's Faces as a Function of Social Salience. Neuropsychopharmacology 37, 1799-1807. Wu, H.Y., Ge, Y., Tang, H.H., Luo, Y.J., Mai, X.Q., Liu, C., 2015. Language modulates brain activity underlying representation of kinship terms. Scientific Reports 5.

Xie, X.Y., Bratec, S.M., Schmid, G., Meng, C., Doll, A., Wohlschlager, A., Finke, K., Forstl, H., Zimmer, C., Pekrun, R., Schilbach, L., Riedl, V., Sorg, C., 2016. How do you make me feel better? Social cognitive emotion regulation and the default mode network. Neuroimage 134, 270280.

Young, L., Dodell-Feder, D., Saxe, R., 2010. What gets the attention of the temporo-parietal junction? An fMRI investigation of attention and theory of mind. Neuropsychologia 48, 26582664. 
bioRxiv preprint first posted online Nov. 8, 2018; doi: http://dx.doi.org/10.1101/465658. The copyright holder for this preprint (which

was not peer-reviewed) is the author/funder, who has granted bioRxiv a license to display the preprint in perpetuity.

It is made available under a CC-BY-NC 4.0 International license.

Zeng, L.L., Shen, H., Liu, L., Wang, L.B., Li, B.J., Fang, P., Zhou, Z.T., Li, Y.M., Hu, D.W., 2012. Identifying major depression using whole-brain functional connectivity: a multivariate pattern analysis. Brain 135, 1498-1507. 
Figure legends

Figure 1 - Functional connectivity with seed in left and right TPJ. Significant brain regions ( $p<0.01$, TFCE corrected) are indicated on the map. The brain regions labeled with yellow/red indicate positive correlation with seed region, while green/blue indicates negative correlation with seed region.

Figure 2 - FC between TPJ and RSNs. A \& B, the FC of left/right TPJ and 14 investigated RSNs for OT and PL group, respectively. The FC were calculated by Pearson correlation between averaged signals across these brain regions. $\mathrm{C}$, $\mathrm{t}$-scores from two sample t-test between TPJ-RSN connectivity. ${ }^{* *} \mathrm{p}<0.01$. Abbreviations: CON, cingulo-opercular network; DMN, default mode network; DAN, dorsal attention network; DSN, dorsal somatomotor network; AN, auditory network; LN, language network, LPN, lateral prefrontal network; IFPN, left frontoparietal network; MPN, medial prefrontal network; rFPN, right frontoparietal network; VAN, ventral attention network; VSN, ventral somatomotor network; VFN, visual foveal network; VPN, visual peripheral network.

Figure 3 - Comparisons between OT and PL group with seed in left and right TPJ, respectively. Seeds in ITPJ and rTPJ are depicted by the yellow dot. For visualization, the brain regions (It-scorel $>3$ ) are indicated on the map. The brain regions labeled with yellow/red indicate OT > PL, while green/blue indicates OT < PL. The peak MNI coordinate regions for each comparison are reported in Table 2. Abbreviations: TPJ, temporo-parietal junction; ITPJ, left temporo-parietal junction; rTPJ, right temporo-parietal junction; OT, oxytocin; PL, plaxebo; TFCE, threshold-free cluster enhancement.

Figure 4 - Empathic concern and TPJ-RECT functional connectivity. The scatter plot displays the linear correlation between the scores of empathic concern ( $x$-axis) and the FC between left TPJ and RECT (y-axis), in OT group (left) and PL group (right). The shaded area represents the $95 \%$ confidence interval of the correlation line. 
Tables, Figures and Supplementary figures

Table 1

Demographic and Clinical Characteristics

\begin{tabular}{lllcc}
\hline & OT group & PL group & t-score $^{\mathrm{a}}$ & p-value \\
\hline age (year) & $22.86(1.57)$ & $22.79(2.27)$ & 0.134 & 0.894 \\
body weight (kg) & $65.87(9.16)$ & $68.21(9.37)$ & -0.970 & 0.336 \\
\hline IRI total scores & $93.83(10.81)$ & $95.10(9.09)$ & -0.478 & 0.634 \\
Perspective taking & $22.79(3.60)$ & $22.03(2.91)$ & 0.883 & 0.381 \\
Fantasy $^{\mathrm{b}}$ & $23.14(5.83)$ & $25.17(5.06)$ & -1.419 & 0.161 \\
Empathic concern $^{\mathrm{b}}$ & $25.83(4.54)$ & $26.21(4.16)$ & -0.332 & 0.741 \\
Personal distress $^{\mathrm{b}}$ & $22.07(3.94)$ & $21.69(4.40)$ & 0.346 & 0.731 \\
\hline
\end{tabular}

Data are expressed as mean $( \pm S D$; standard deviation); $n=30$ for oxytocin group; $n=29$ for placebo group.

Abbreviations: OT, oxytocin; PL, placebo; IRI, Interpersonal Reactivity Index;

at-ttest, two tailed.

${ }^{b}$ Data missing from one OT group $(n=29)$. 
bioRxiv preprint first posted online Nov. 8, 2018; doi: http://dx.doi.org/10.1101/465658. The copyright holder for this preprint (which was not peer-reviewed) is the author/funder, who has granted bioRxiv a license to display the preprint in perpetuity.

It is made available under a CC-BY-NC 4.0 International license.

Figure 1 - Functional connectivity with seed in left and right TPJ. Significant brain regions $(p<0.01$, TFCE corrected) are indicated on the map. The brain regions labeled with yellow/red indicate positive correlation with seed region, while green/blue indicates negative correlation with seed region.

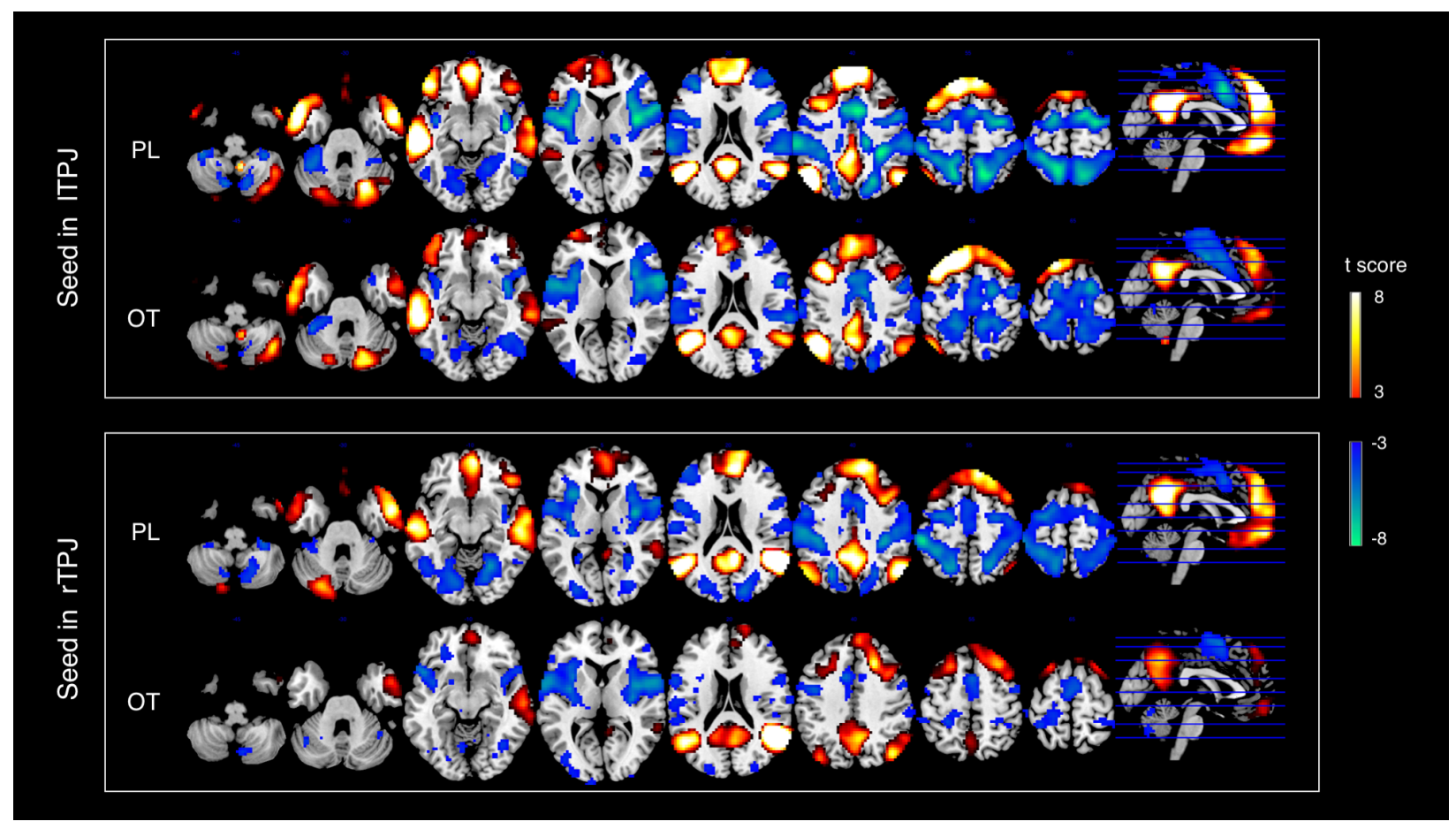


Figure 2 - FC between TPJ and RSNs.

$A$ and $B$, the FC of left/right TPJ and 14 investigated RSNs for OT and PL group, respectively. The FC were calculated by Pearson correlation between averaged signals across these brain regions. C, t-scores from two sample t-test between TPJ-RSN connectivity. ${ }^{* *} \mathrm{p}<0.01$ with FDR correction. Abbreviations: CON, cingulo-opercular network; DMN, default mode network; DAN, dorsal attention network; DSN, dorsal somatomotor network; AN, auditory network; LN, language network, LPN, lateral prefrontal network; IFPN, left frontoparietal network; MPN, medial prefrontal network; rFPN, right frontoparietal network; VAN, ventral attention network; VSN, ventral somatomotor network; VFN, visual foveal network; VPN, visual peripheral network.

A

\begin{tabular}{|c|c|c|c|c|c|c|c|c|c|c|c|c|c|}
\hline \multicolumn{14}{|c|}{ OT } \\
\hline ITPJ -0.45 & 0.45 & -0.12 & -0.38 & -0.32 & 0.58 & 0.05 & 0.29 & 0.13 & 0.10 & 0.30 & -0.23 & -0.18 & -0.13 \\
\hline rTPJ -0.36 & 0.54 & 0.16 & -0.23 & -0.27 & 0.09 & 0.03 & 0.05 & 0.09 & 0.14 & 0.51 & -0.21 & -0.17 & -0.06 \\
\hline
\end{tabular}

B

$\mathrm{PL}$

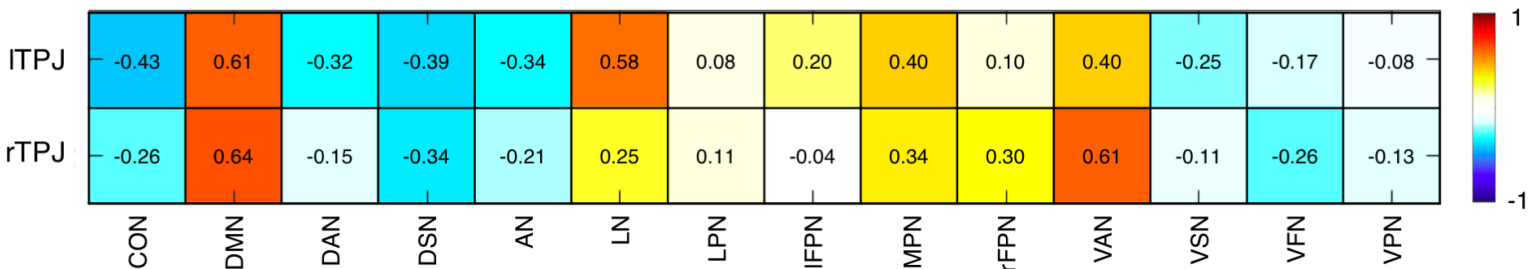

$\mathrm{C}$

OT v.s. PL

tscore

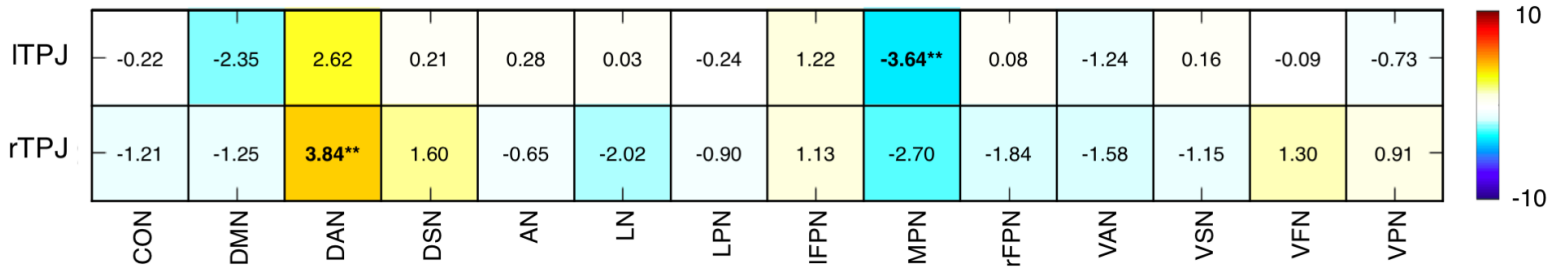


Figure 3 - Comparisons between OT and PL group with seed in left and right TPJ, respectively. Seeds in ITPJ and rTPJ are depicted by the yellow dot. For visualization, the brain regions (threshold at It-scorel>3) are plotted over a representative cortical surface in dorsal, lateral, and medial views. The brain regions labeled with yellow/red indicate OT > PL, while green/blue indicates OT < PL. The peak MNI coordinate regions for each comparison are reported in Table 2. Abbreviations: TPJ, temporo-parietal junction; ITPJ, left temporo-parietal junction; rTPJ, right temporo-parietal junction; OT, oxytocin; PL, plaxebo; TFCE, threshold-free cluster enhancement.

ITPJ

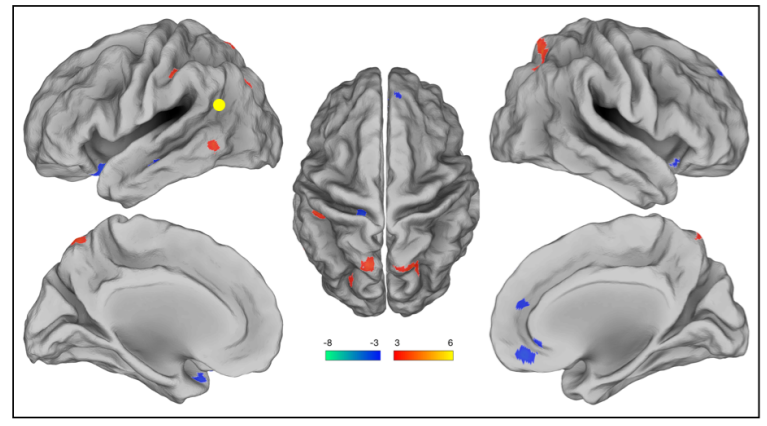

rTPJ

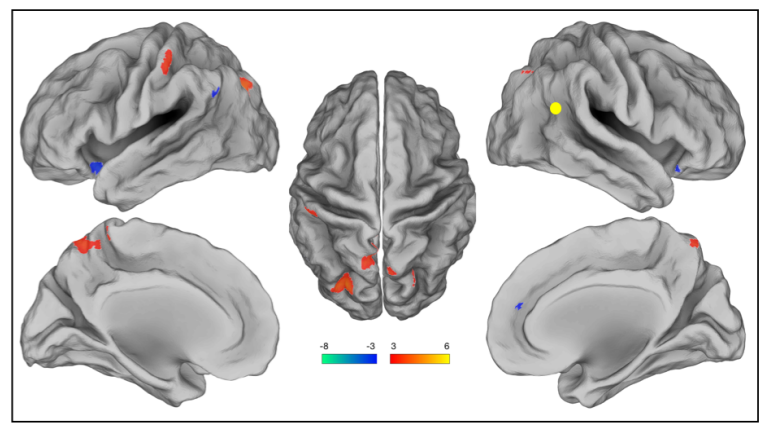

Table 2 - Results of between-subjects factor comparisons on the functional connectivity with TPJ4

\begin{tabular}{lccccc}
\hline Contrast & Connectivity & T-score & X (MNI) & $\mathrm{Y}(\mathrm{MNI})$ & $\mathrm{Z}(\mathrm{MNI})$ \\
\hline OT > PL & ITPJ-IIPL & 4.36 & 43 & -32 & 37 \\
& rTPJ-IPrec & 3.82 & -15 & -59 & 62 \\
& rTPJ-rSPL & 3.96 & 21 & -58 & 50 \\
& rTPJ-rPrec & 3.55 & 7 & -68 & 64 \\
& rTPJ-IMOG & 4.38 & -25 & -76 & 37 \\
\hline OT <PL & ITPJ-rRect & 4.35 & 5 & 46 & -14 \\
& rTPJ-IInsula & 3.82 & -28 & 20 & -15 \\
& rTPJ-IIFG & 7.00 & -56 & 28 & 2 \\
\hline
\end{tabular}

Peak MNI coordinates and t-scores for between-subjects factor comparison between OT and PL. Abbreviations: ITPJ, left temporo-parietal junction; rTPJ, right temporo-parietal junction; IIPL, left inferior parietal lobule; rRect, right rectus; IPrec, left Precuneus; rSPL, right superior parietal lobe; IMOG, left middle occipital gyrus; IInsula, Left insula; IIFG, inferior frontal gyrus. 
Table 3 - TPJ functional connectivity and the corresponding weight for SVM classification

\begin{tabular}{lc}
\hline Connectivity & weight \\
\hline ITPJ-rRect & $0.3205(0.0015)$ \\
ITPJ-IIPL & $-0.2769(0.0019)$ \\
rTPJ-IPrec & $0.1104(0.0027)$ \\
rTPJ-rSPL & $-0.2036(0.0018)$ \\
rTPJ-rPrec & $-0.0291(0.0023)$ \\
rTPJ-IMOG & $-0.2963(0.0018)$ \\
rTPJ-IInsula & $0.2761(0.0019)$ \\
rTPJ-IIFG & $0.1079(0.0023)$ \\
\hline
\end{tabular}

Data are expressed as mean ( \pm SEM; standard error of the mean); $\mathrm{n}=59$ for leave-oneout validation; accuracy $=74.58 \%$; sensitivity $=73.33 \%$; specificity $=75.86 \%$.

Figure 4 - Empathic concern and TPJ-RECT functional connectivity. The scatter plot displays the linear correlation between the scores of empathic concern ( $x$-axis) and the FC between left TPJ and RECT ( $y$-axis), in OT group (A) and PL group (B). The shaded area represents the $95 \%$ confidence interval of the correlation line. $\mathrm{N}=29$ for OT group; $\mathrm{N}=29$ for $\mathrm{PL}$ group.

A

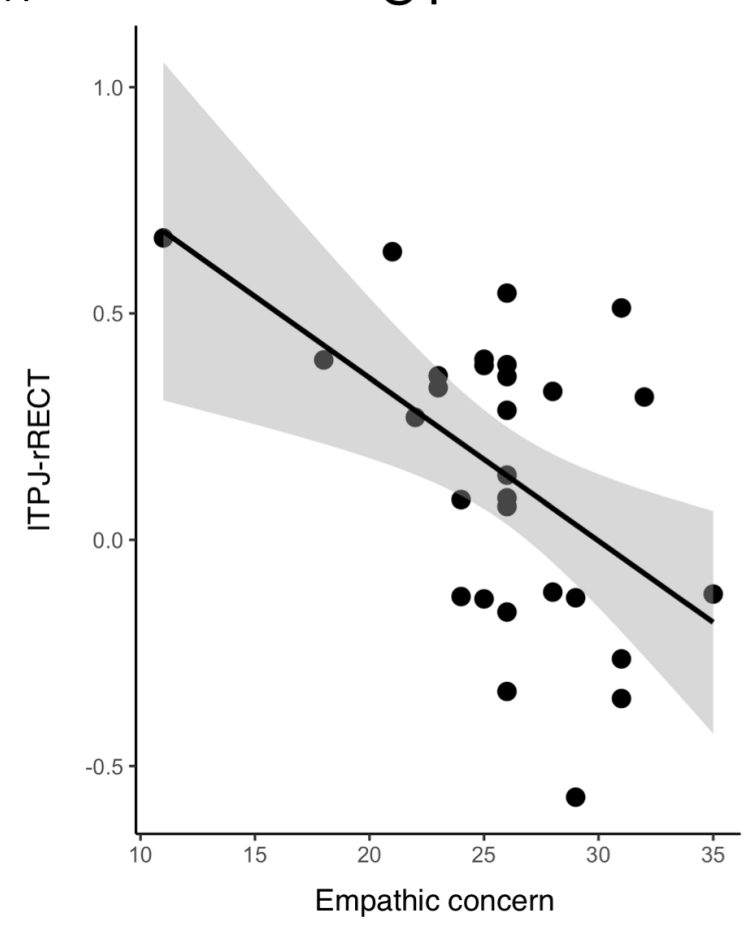

B

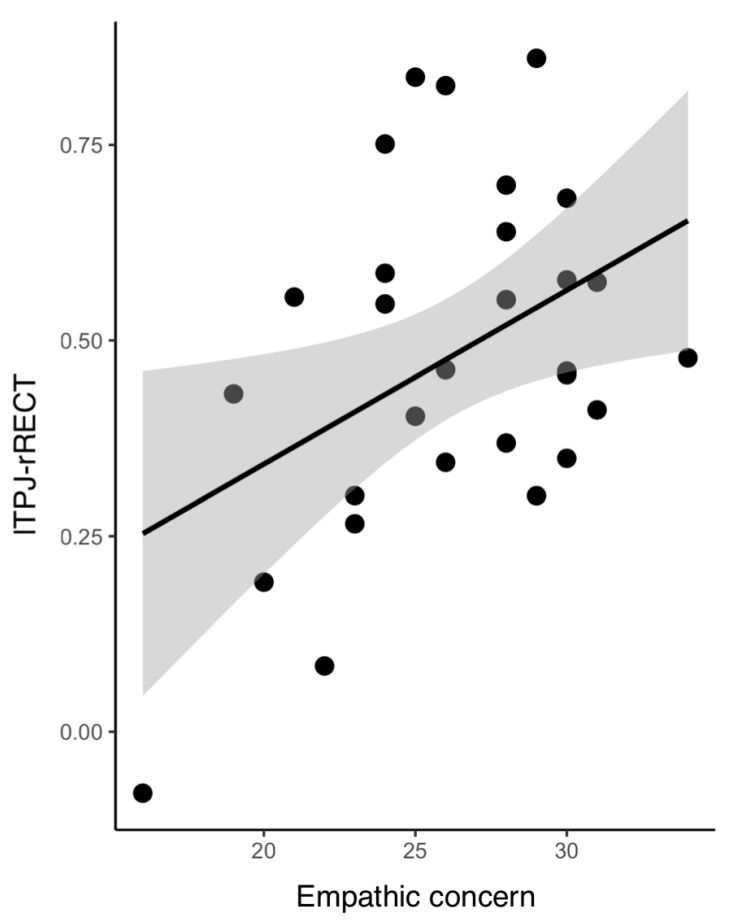


Supplementary Figure 1 - 14 RSNs extracted from OT group and PL group. The RSN maps were selected from IC maps which extracted from ICA analysis on BOLD signal time series for each individual, by a template-matching strategy. The group-level RSN maps from OT group and PL group were presented. Surprisingly, the spatial maps showed no topological differences and no brain regions survived from TFCE correction, which implies that OT has no effects on the topology of large-scale RSNs.

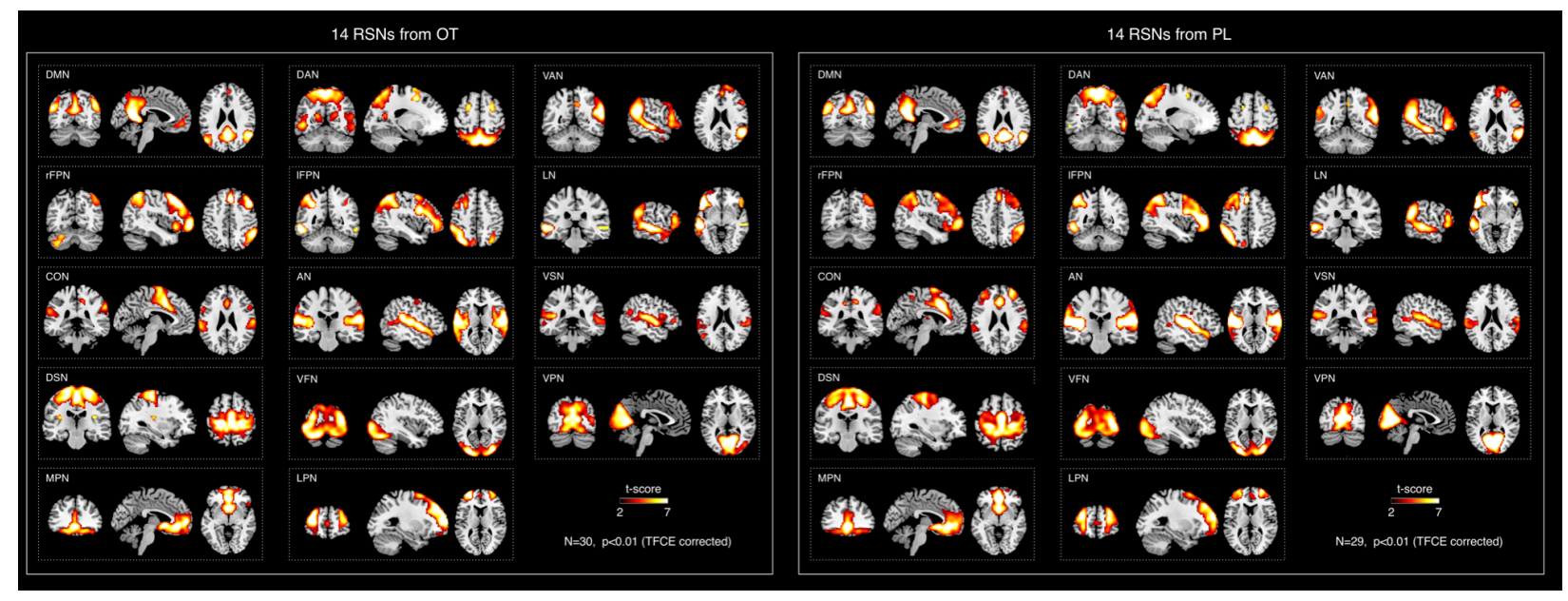

Supplementary Figure 2 - Boxplot of functional connectivity between the identified eight pairs of regions.
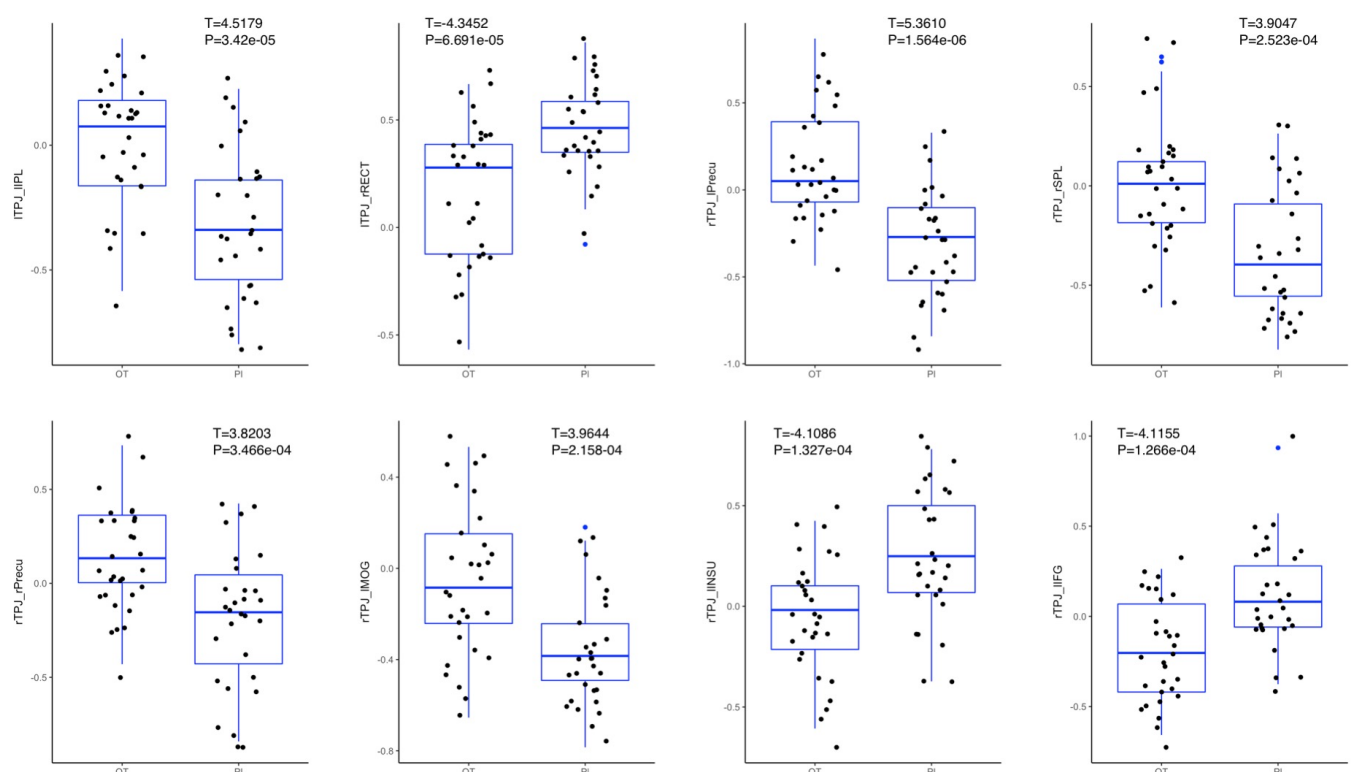
Supplementary Figure 3 - The relationship between perspective taking and the TPJ connectivity. The brain regions correlated (threshold at $r>0.5$, labeled with yellow/red) or anitcorrelated (threshold at $r<-0.5$, labeled with green/blue) with perspective taking scale are indicated on the map.

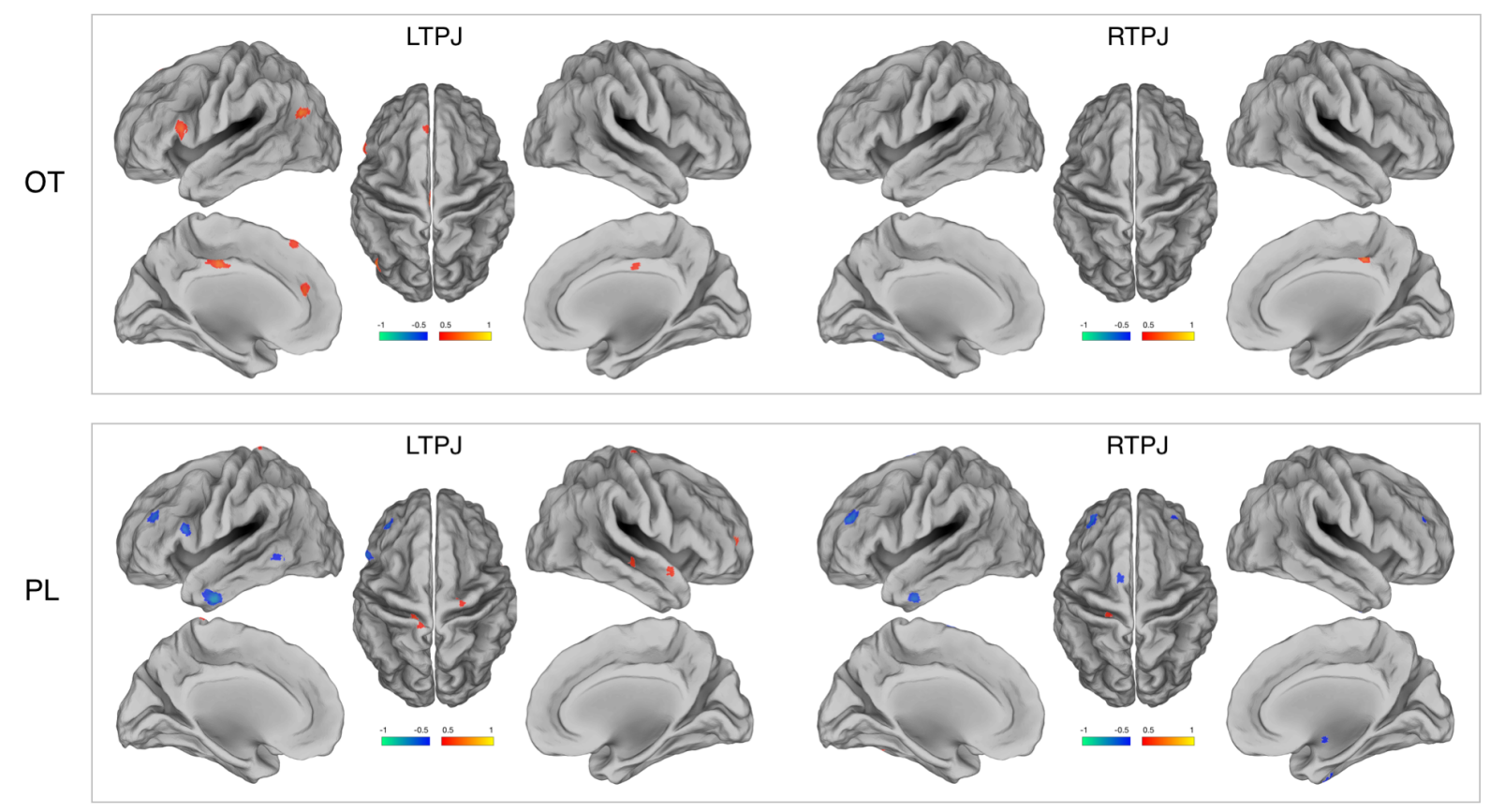


bioRxiv preprint first posted online Nov. 8, 2018; doi: http://dx.doi.org/10.1101/465658. The copyright holder for this preprint (which was not peer-reviewed) is the author/funder, who has granted bioRxiv a license to display the preprint in perpetuity.

It is made available under a CC-BY-NC 4.0 International license.

Supplementary Figure 4 - The relationship between fantasy scale and TPJ connectivity. The brain regions correlated (threshold at $r>0.5$, labeled with yellow/red) or anitcorrelated (threshold at $r<-0.5$, labeled with green/blue) with fantasy scale are indicated on the map.

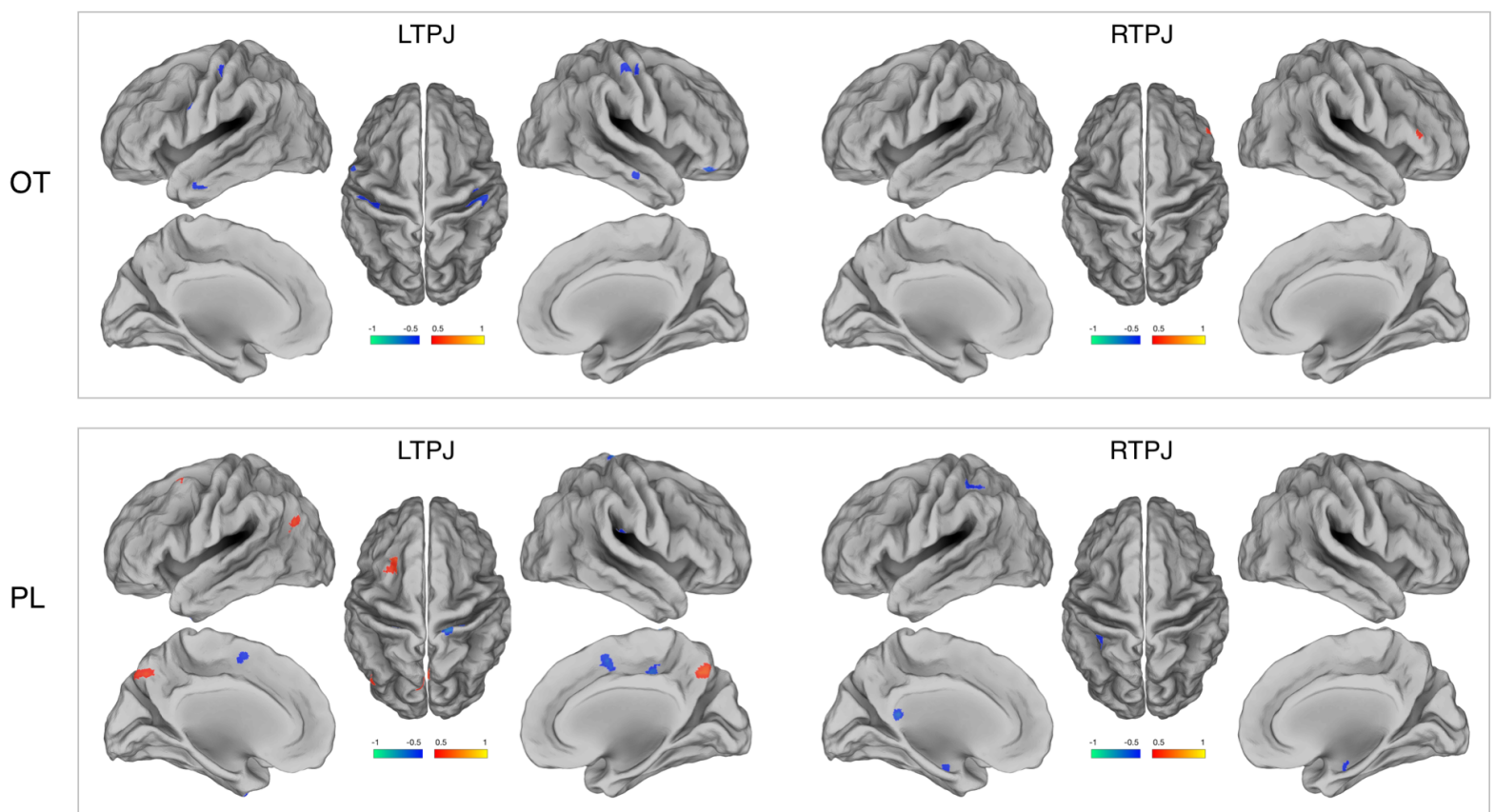


bioRxiv preprint first posted online Nov. 8, 2018; doi: http://dx.doi.org/10.1101/465658. The copyright holder for this preprint (which was not peer-reviewed) is the author/funder, who has granted bioRxiv a license to display the preprint in perpetuity.

It is made available under a CC-BY-NC 4.0 International license.

Supplementary Figure 5 - Empathic concern mediates the TPJ connectivity. The brain regions correlated $(r>0.5$, labeled with yellow/red) or anitcorrelated $(r<-0.5$, labeled with green/blue) with empathic concern scale are indicated on the map.
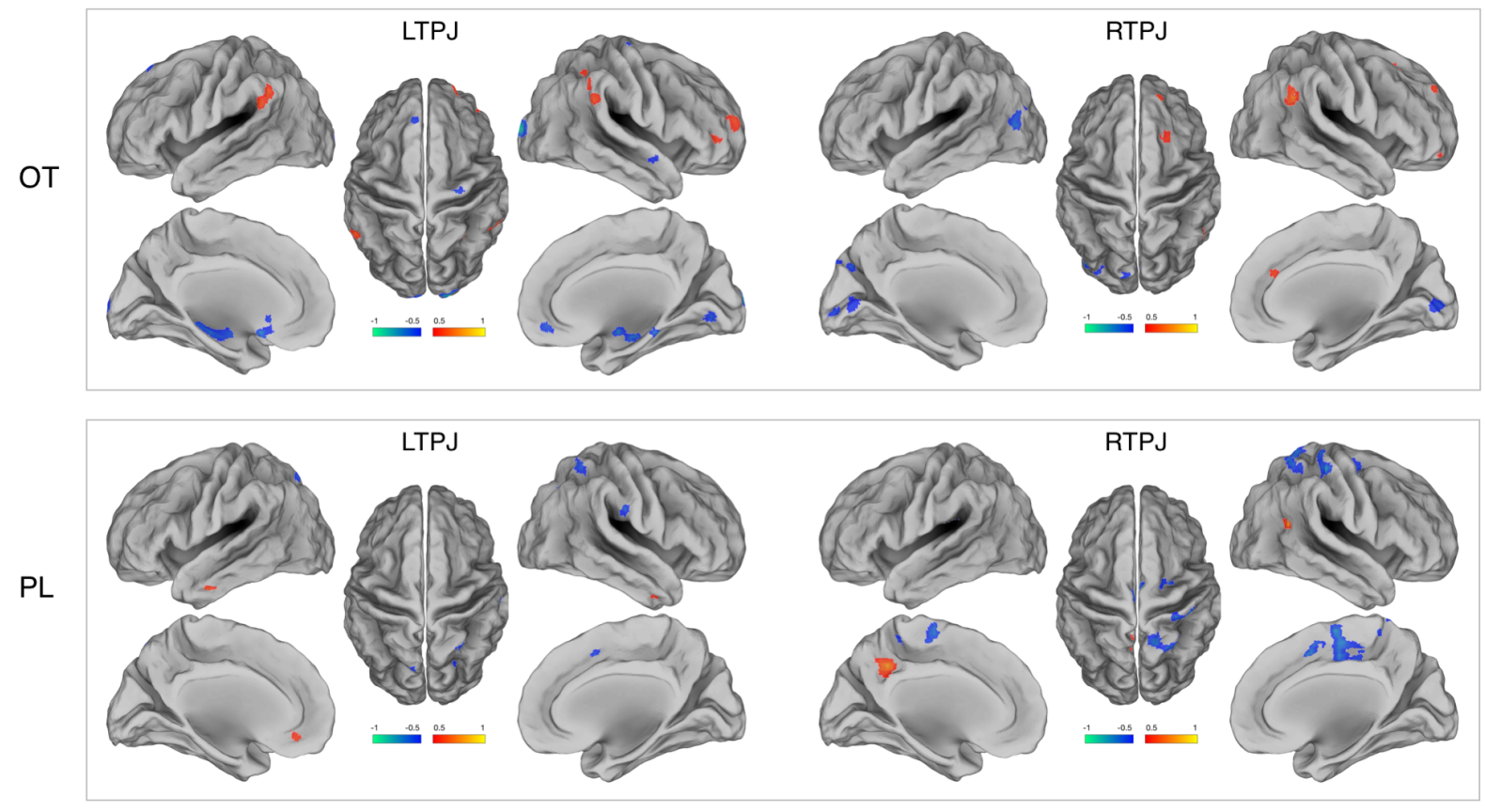

Supplementary Figure 6 - Personal distress mediates the TPJ connectivity. The brain regions correlated $(r>0.5$, labeled with yellow/red) or anitcorrelated $(r<-0.5$, labeled with green/blue) with personal distress scale are indicated on the map.
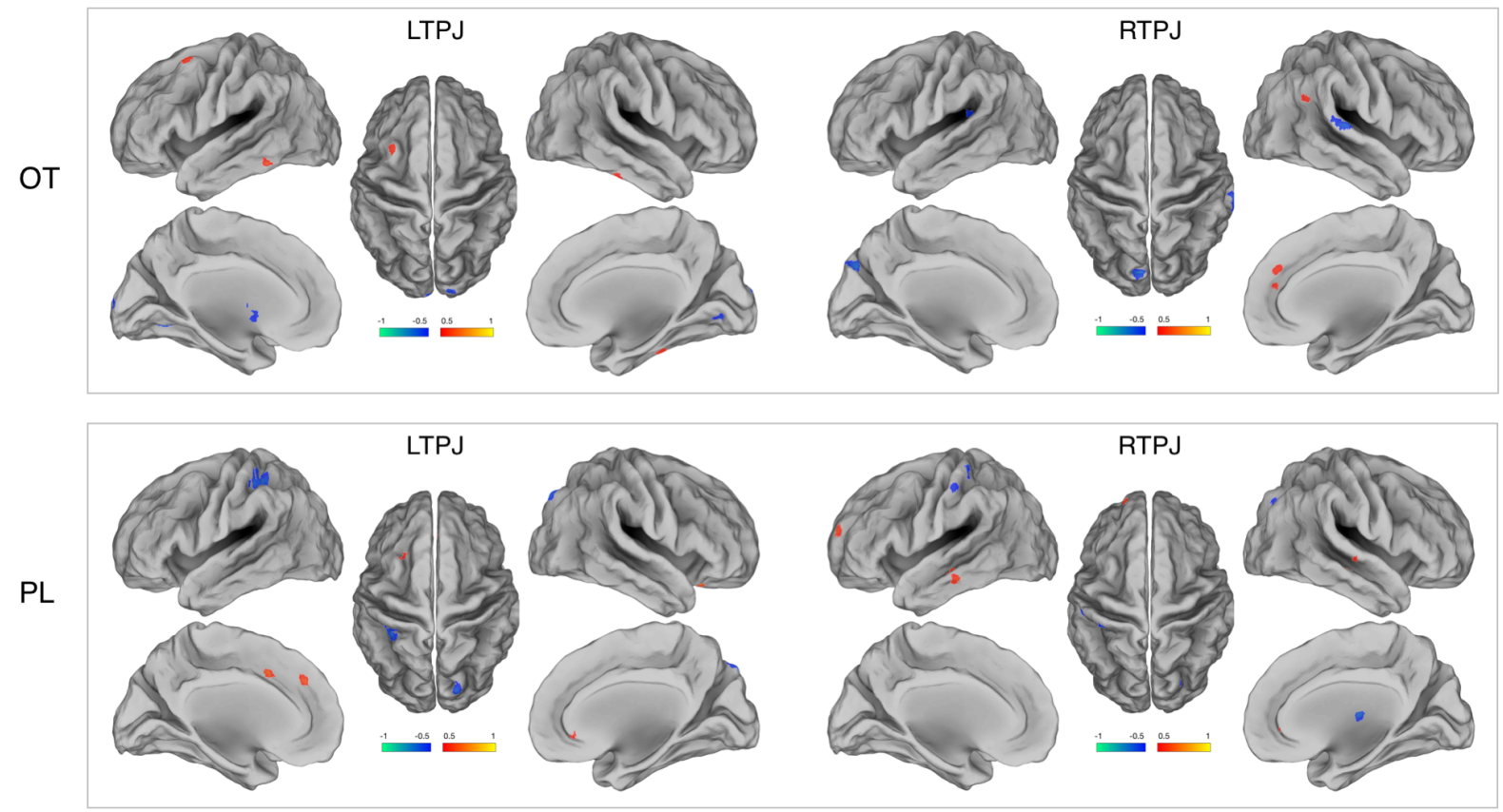